\title{
Neighbourhood communalities and Ultra-High Risk psychosis: an emergent literature
}

\author{
Kirkbride JB, ${ }^{a *}$ Perez J, ${ }^{b}$ Jones $\mathrm{PB}^{\mathrm{b}}$ \\ a PsyLife Research Group, Division of Psychiatry, UCL, London, W1T 7NF \\ ${ }^{\mathrm{b}}$ Department of Psychiatry, University of Cambridge, Cambridge, CB2 OSZ
}

*Corresponding author: j.kirkbride@ucl.ac.uk, PsyLife Research Group, Division of Psychiatry, UCL, $6^{\text {th }}$ Floor, 149 Tottenham Court Road, London, W1T 7NF, UK, +44 (0) 2076799297

Key words: ultra-high risk; early intervention; psychosis; environment; social determinants

\section{Letter to the Editor}

The work of O'Donoghue et al. (2015) provides a welcome contribution to the emergent field exploring the association between the wider neighbourhood environment and the rate of ultra-high risk [UHR] states for psychosis.

A large literature already demonstrates that the incidence of psychotic disorders, and most strongly non-affective psychotic disorders, is raised in populations exposed to urban living (Kirkbride et al., 2012). However, epidemiological studies conducted on the basis of residency close to the onset of psychosis have not been able to exclude the possibility that reverse causation, or social drift, explains such associations, whereby people in the prodromal phase of schizophrenia are more likely to move into more urban areas, often characterised by greater levels of social disadvantage (or less likely to be able to move out of them). While findings which show strong dose-response gradients between urban birth and later psychosis risk provide some evidence against the possibility of social drift (Mortensen et al., 1999), a further way to triangulate this hypothesis is to study the neighbourhood characteristics of people who meet UHR criteria for psychosis. Measuring the association between exposure to urban environments during or before the prodromal phase of psychotic illness, potentially allows us to get a better grip on the direction of causality between urban residency and later psychosis risk.

Studies such as that by O'Donoghue et al. (2015) are thus a potential boon to aetiological understanding of psychotic disorders. They also provide important information for mental health service planners, who may be interested in understanding which populations are most likely to express need for psychiatric care, which operates over a strong social and spatial gradient (Kirkbride et al., 2013). In their paper, based on a sample of 166 people who met UHR criteria living in a defined catchment area in Melbourne, Australia, O'Donoghue et al. (2015) show that the UHR incidence varies by deprivation, with a weak linear trend towards higher UHR rates in more deprived areas (RR: $1.51 ; 95 \% \mathrm{Cl}: 0.93-2.53 ; \mathrm{p}=0.08$ ). However, further inspection of this data suggests that (a) absolute rates were higher in the most affluent parts of their study area compared with the most deprived, and (b) the weak linear trend smooths over apparent non-linearity in the relationship between deprivation and UHR incidence.

O'Donoghue et al. (2015) suggest that their results "[cast doubt] on recent findings of a higher distribution of UHR cases in more affluent neighbourhoods (Kirkbride et al., 201[5])". By contrast, we suggest that their findings are, in fact, consistent with those observed in our sample in the East of England (Kirkbride et al., 2015). Both studies, for example, used identical criteria to define UHR groups. Our multivariable results, based on a population-based sample, did indeed show that the odds of receiving UHR status (versus control) were increased in more affluent (or less deprived) neighbourhoods. On one level this is consistent with O'Donogue et al.'s (2015) observation of higher 
UHR rates in their most vs least affluent areas.

On another level, the data from both papers suggests that the relationship between deprivation and UHR status is probably non-linear, and such a finding is consistent with some evidence on the incidence of psychotic disorders (Croudace et al., 2000). In our paper, the negative association between deprivation per se and UHR for psychosis only appeared after adjustment for other neighbourhood-level characteristics, including the proportion of single-parent households, which showed a strong trend towards increased risk of UHR status (RR: $1.59 ; 95 \% \mathrm{Cl}: 0.99-2.57 ; p=0.056$ ). Given that single-parent household status was highly correlated with neighbourhood deprivation $(r=$ 0.66 ) in our sample, we suggest that "[h]aving controlled for this, the counterintuitive negative association between multiple deprivation and psychosis proneness might indicate a nonlinear relationship." (Kirkbride et al., 2015). Interpreted in this light, our findings also accord with O'Donoghue et al. (2015), given the presence of non-linearity between deprivation and the rate of UHR groups in their data (see their Table 1). Both papers were based on relatively small epidemiological samples of people at UHR for psychosis, making it important to replicate these findings in further, sufficiently-powered studies. Given the high levels of heterogeneity which likely exist in UHR samples (Fusar-Poli et al., 2016), careful delineation of the group(s) at risk will also be required in future studies.

We hope that our reply comment adds some further insight into the results from both O'Donoghue et al (2015) and Kirkbride et al. (2015), which represent an emergent, important area for further research. Our conclusion remains the same: taken together, these observations support the possibility that people with increased psychosis proneness are exposed to more adverse social environments during the at-risk period. This has potentially important implications for both aetiology and public mental health.

\section{Funding body agreements and policies}

James Kirkbride is supported by a Sir Henry Dale Fellowship jointly funded by the Wellcome Trust and the Royal Society (grant number: 101272/Z/13/Z). Peter Jones directs the NIHR Collaboration for Leadership in Applied Health Research and Care (CLAHRC) for Cambridgeshire \& Peterborough and is supported by NIHR grant RP-PG-0606-1335.

\section{Role of funding source}

The funding sources had no involvement in study design; in the collection, analysis, and interpretation of data; in the writing of the report; or in the decision to submit the paper for publication.

\section{References}

Croudace, T.J., Kayne, R., Jones, P.B., Harrison, G.L., 2000. Non-linear relationship between an index of social deprivation, psychiatric admission prevalence and the incidence of psychosis. Psychol. Med. 30, 177-185.

Fusar-Poli, P., Cappucciati, M., Borgwardt, S., Woods, S.W., Addington, J., Nelson, B., Nieman, D.H., Stahl, D.R., Rutigliano, G., Riecher-Rössler, A., Simon, A.E., Mizuno, M., Lee, T.Y., Kwon, J.S., Lam, M.M.L., Perez, J., Keri, S., Amminger, P., Metzler, S., Kawohl, W., Rössler, W., Lee, J., Labad, J., Ziermans, T., An, S.K., Liu, C.-C., Woodberry, K.A., Braham, A., Corcoran, C., McGorry, 
P., Yung, A.R., McGuire, P.K. 2016. Heterogeneity of Psychosis Risk Within Individuals at Clinical High Risk. JAMA Psychiatry 73, 113. doi:10.1001/jamapsychiatry.2015.2324

Kirkbride, J.B., Errazuriz, A., Croudace, T.J., Morgan, C., Jackson, D., Boydell, J., Murray, R.M., Jones, P.B., 2012. Incidence of Schizophrenia and Other Psychoses in England, 1950-2009: A Systematic Review and Meta-Analyses. PLoS One 7, e31660. doi:10.1371/journal.pone.0031660

Kirkbride, J.B., Jackson, D., Perez, J., Fowler, D., Winton, F., Coid, J.W., Murray, R.M., Jones, P.B., 2013. A population-level prediction tool for the incidence of first-episode psychosis: translational epidemiology based on cross-sectional data. BMJ Open 3. doi:10.1136/bmjopen2012-001998

Kirkbride, J.B., Stochl, J., Zimbrón, J., Crane, C.M., Metastasio, A., Aguilar, E., Webster, R., Theegala, S., Kabacs, N., Jones, P.B., Perez, J., 2015. Social and spatial heterogeneity in psychosis proneness in a multilevel case-prodrome-control study. Acta Psychiatr. Scand. 132, 283-292. doi:10.1111/acps.12384

Mortensen, P.B., Pedersen, C.B., Westergaard, T., Wohlfahrt, J., Ewald, H., Mors, O., Andersen, P.K., Melbye, M., 1999. Effects of family history and place and season of birth on the risk of schizophrenia. N. Engl. J. Med. 340, 603-608.

O’Donoghue, B., Yung, A.R., Wood, S., Thompson, A., Lin, A., McGorry, P., Nelson, B., 2015. Neighbourhood characteristics and the rate of identification of young people at ultra-high risk for psychosis. Schizophr. Res. 169, 214-216. doi:10.1016/j.schres.2015.09.002 